\title{
An $\mathrm{H} \alpha$ study of the kinematics of NGC $3627^{\star}$
}

\author{
L. Chemin ${ }^{1}$, V. Cayatte ${ }^{1}$, C. Balkowski ${ }^{1}$, M. Marcelin ${ }^{2}$, P. Amram ${ }^{2}$, W. van Driel ${ }^{1}$, and H. Flores ${ }^{1}$ \\ 1 Observatoire de Paris, GEPI, UMR 8111, associé au CNRS et à l'Université Paris VII, 5 place J. Janssen, \\ 92195 Meudon, France \\ 2 Observatoire Astronomique Marseille-Provence, 2 place Le Verrier, 13248 Marseille, France
}

Received 24 September 2002 / Accepted 15 April 2003

\begin{abstract}
We report here on the first observations in the $\mathrm{H} \alpha$ line to investigate the kinematics of the spiral galaxy NGC 3627 , the brightest member of the Leo Triplet. The velocity field observed at high angular resolution shows signatures of non-circular motion. They can be attributed to dynamical perturbations due to the bar and to external interactions. In particular, we confirm the presence of an inner ring recently detected in CO observations (Regan et al. 2002). We find a corotation radius significantly smaller than that derived in previous studies and we show that the inner ring location is consistent with the ultra-harmonic resonance induced by the bar pattern speed. We also show that a warped tilted-ring model can be fitted to the outer parts of the southern, receding half of the disc, which is inclined by some $15^{\circ}$ relative to the inner parts of the galaxy.
\end{abstract}

Key words. galaxies: individual: NGC 3627 - galaxies: kinematics and dynamics - galaxies: structure

\section{Introduction}

NGC 3627 (Messier 66) is a spiral galaxy classified as SABb in the RC3 catalogue (de Vaucouleurs et al. 1991), showing signatures of a Liner/Seyfert 2-type nuclear activity in its spectrum (Ho et al. 1997). The optical broad-band image of NGC 3627 reveals a pronounced spiral pattern with heavy dust lanes, indicating a strong density wave action. The pattern is asymmetric with respect to the major axis. Moreover, this image shows a perturbed morphology of the eastern arm which appears to have been displaced from the plane of the galaxy. With NGC 3623 and NGC 3628, it forms the Leo Triplet. Low resolution HI mapping of the Triplet (Rots 1978; Haynes et al. 1979) has revealed signs of past interactions between the two largest spirals, NGC 3627 and NGC 3628: a tail between these galaxies, a long plume extending about $50^{\prime}$ to the east of NGC 3628 and a non-corotating component in the eastern outskirts of the HI disc of NGC 3627. Using a restricted threebody code, Toomre (1977) and Rots (1978) have proposed a model of a retrograde encounter of NGC 3627 moving along a parabolic orbit around NGC 3628. HI and CO-line studies of NGC 3627 (Zhang et al. 1993; Reuter et al. 1996) show clear asymmetries in the velocity field of the atomic and molecular gas.

A high resolution total power and polarization VLA mapping of this galaxy in the radio continuum (Soida et al. 2001) has revealed the existence of two components of the magnetic

Send offprint requests to: $\mathrm{L}$. Chemin, e-mail: Laurent.Chemin@obspm.fr

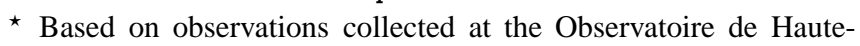
Provence (France). field: the interarm smoothly distributed field and the S-shaped component related to the bar and spiral arms. These components sometimes meet each other at a very different pitch angle (defined as the angle between the local magnetic field orientation and the azimuthal direction). While in the western arm the S-shaped component follows well the density wave flows and the dust lane, in the south-eastern disc it crosses the optical arm at a high angle, despite strong gas compression effects as indicated by a heavy dust lane. High spatial resolution observations of the kinematics of the ionized gas are needed to understand the origin of this peculiar magnetic field behaviour in the galaxy.

Although it was this problem that initially motivated the present study, we want to focus here only on the kinematical properties of the ionized gas in NGC 3627, which was observed as part of the $\mathrm{GHASP}^{1}$ project (Garrido et al. 2002, 2003), a survey of the ionized hydrogen kinematics of about 200 nearby galaxies by Fabry-Perot interferometry. We want to examine possible gas kinematic perturbations in the galactic disc and to present the supporting observational data needed for future 3D dynamo simulations of the radio polarization and of the magnetic field (see e.g. Otmianowska-Mazur et al. 2002). We will address the relationship between the gas flows and the peculiar magnetic field in a forthcoming paper.

Moreover, in a recent paper, Regan et al. (2002) noted the presence of an unseen inner ring in NGC 3627 from BIMA CO-line observations at a resolution of $\sim 6$ " (Regan et al. 1999). Rings are usually observed in barred galaxies and are easily revealed on optical photographic plates because intense star

\footnotetext{
${ }^{1}$ Gassendi H Alpha survey of SPirals.
} 
formation which take place in them leads to a high intensity contrast with respect to their surroundings. However, surprisingly, NGC 3627 has never been classified as a ringed galaxy. Regan et al. (2002) pointed out that the combination of a high inclination with the presence of intense star formation throughout the bar region makes the detection of the inner ring difficult from optical images.

According to the classical dynamical scenario in a barred potential, an inner ring forms preferentially at ultra-harmonic resonant orbits (see Buta \& Combes 1996, and references therein) as confirmed by observations (see e.g. Buta et al. 2001). If the presence of an inner ring is confirmed in NGC 3627 from the $\mathrm{H} \alpha$ observations, the knowledge of its location and of the disc rotation curve will constrain the pattern speed of the bar.

Section 2 describes the observations and the data reduction techniques, Sect. 3 presents the distribution of the ionized gas. In Sect. 4 we analyze the observed kinematics of the ionized gas. The discussion of the results is presented in Sect. 5, the main conclusions are summarized in Sect. 6 .

\section{Observations and data reduction}

\subsection{Observations}

We observed NGC 3627 in the $\mathrm{H} \alpha$ line in March 2000 using the GHASP instrument ${ }^{2}$, a focal reducer with a scanning FabryPerot interferometer and a bi-dimensional photon counting detector, mounted on the $1.93 \mathrm{~m}$ telescope of the Observatoire de Haute-Provence (OHP) in France.

Using the old configuration of GHASP, the field of view was $4.1 \times 4.1$ on the sky, covered by $256 \times 256$ pixels of $0^{\prime \prime} .96 \times$ $0^{\prime \prime} .96$. The interference filter was centered at $\lambda=6576.00 \AA$ and has a $F W H M$ of $11.00 \AA$. The $p=793$ interference order provided a Free Spectral Range $(F S R=c / p)$ of $378 \mathrm{~km} \mathrm{~s}^{-1}$ (or $8.3 \AA$ at the rest wavelength of the $\mathrm{H} \alpha$ line, $6562.78 \AA$ ). The FSR was scanned through 24 channels, resulting in a spectral sampling of $15.8 \mathrm{~km} \mathrm{~s}^{-1}$. The total exposure time for the galaxy was about 2 hours and the seeing was about $3^{\prime \prime}$. As the angular size of the galaxy exceeds the field of view of the GHASP instrument, the southernmost part of the western spiral arm and the northernmost tip of the eastern arm could not be observed.

Table 1 gives the journal of the observations and Table 2 summarizes the basic properties of NGC 3627, as well as some parameters deduced from this study.

\subsection{Data reduction}

The data reduction procedure for the raw $256 \times 256 \times 24$ data cube has been described in several papers (e.g. Marcelin et al. 1987; Amram et al. 1992). First, using a calibration data cube obtained with a Neon lamp ( $\lambda=6598.95 \AA)$ before and after the observation of the galaxy, a wavelength calibrated map is made, in which each pixel has its own wavelength reference (the phase map). The calibrated data are then smoothed in order

\footnotetext{
${ }^{2}$ For further details on the GHASP project and instrument, see http://www-obs.cnrs-mrs.fr/interferometrie/ GHASP/ghasp.html
}

Table 1. Journal of the Fabry-Perot observations.

\begin{tabular}{ll}
\hline \hline Observations & \\
\hline Telescope & OHP 1.93 m \\
Equipment & GHASP @ Cassegrain focus \\
Date & March 2000 \\
Seeing & $\sim 3^{\prime \prime}$ \\
\hline Interference Filter & \\
\hline Central Wavelength & $6576 \AA$ \\
FWHM & $11 \AA$ \\
Transmission & 0.74 \\
\hline Calibration & \\
\hline Neon Comparison light & $\lambda 6598.95 \AA$ \\
\hline Fabry-Perot & \\
\hline Interference Order & $793 @ 6562.78 \AA$ \\
Free Spectral Range at H $\alpha$ & $\sim 380 \mathrm{~km} \mathrm{~s}^{-1}$ \\
Finesse at H $\alpha$ & 10 \\
Spectral resolution at H $\alpha$ & 18750 at the sample step \\
\hline Sampling & \\
\hline Number of Scanning Steps & 24 \\
Sampling Step & $0.35 \AA\left(16 \mathrm{~km} \mathrm{~s}^{-1}\right)$ \\
Total Field & $4.1^{\prime} \times 4.1^{\prime}\left(256 \times 256 \mathrm{px}^{2}\right)$ \\
Pixel Size & $0.96^{\prime \prime}$ \\
\hline Exposures times & \\
\hline Total exposure & 2 hours \\
Elementary scanning & $15 \mathrm{~s} \mathrm{per} \mathrm{channel}$ \\
exposure time & \\
\hline & \\
\hline
\end{tabular}

Table 2. Basic parameters of NGC 3627.

\begin{tabular}{|c|c|}
\hline Right ascension (J2000) & $11^{\mathrm{h}} 20^{\mathrm{m}} 15^{\mathrm{s}}$ \\
\hline Declination (J2000) & $+12^{\circ} 59^{\prime} 30^{\prime \prime}$ \\
\hline Classification $^{a}$ & $\mathrm{SAB}(\mathrm{s}) \mathrm{b}$ \\
\hline Nucleus $^{b}$ & LINER/Sy2 \\
\hline $\operatorname{Distance}^{c}(\mathrm{Mpc})$ & 11.1 \\
\hline Linear scale $\left(\mathrm{pc} \operatorname{arcsec}^{-1}\right)$ & 54 \\
\hline Kinematical inclination ${ }^{e}\left({ }^{\circ}\right)$ & $65 \pm 5$ \\
\hline Kinematical position angle ${ }^{e}\left(^{\circ}\right)$ & $170 \pm 5$ \\
\hline Heliocentric systemic velocity $\left(\mathrm{km} \mathrm{s}^{-1}\right)$ & $727 \pm 5$ \\
\hline Optical major axis diameter $D_{25}^{d}\left(^{\prime}\right)$ & 8.7 \\
\hline Mean $\mathrm{H} \alpha$ bar position angle $\left(^{\circ}\right)$ & $155 \pm 5$ \\
\hline Bar radius ${ }^{f}(")$ & 49 \\
\hline
\end{tabular}

a de Vaucouleurs et al. (1991).

${ }^{\mathrm{b}}$ Ho et al. (1997).

c Saha et al. (1999).

d Paturel et al. (1991).

e This paper.

${ }^{\mathrm{f}}$ Das et al. (2003).

to increase the signal-to-noise ratio in the faintest parts of the galaxy. This was done by applying a Gaussian smoothing to the $\mathrm{H} \alpha$ profiles, as well as a spatial smoothing in $X$ and $Y$ with a $F W H M$ of 3 pixels.

The average night sky emission line is then subtracted from the entire data cube. The sky line consists of two $\mathrm{OH}$ lines at $6577.18 \AA$ and at $6577.38 \AA$, which are too close to each other to be separated by the interferometer. 
For each pixel, the signal is the sum of an intensity level which remains almost constant over the 24 channels (the continuum) and of the $\mathrm{H} \alpha$ emission line. To avoid contamination by the emission line, which is relatively wide compared to a limited number of channels, the continuum intensity level is taken as the mean of the 3 lowest intensity channels.

A map of the integrated $\mathrm{H} \alpha$ line intensity distribution is made by summing over all channels where the signal found in the emission line is above the continuum level. The heliocentric radial velocity for a given pixel is the position of the barycentre of the line. We thus obtain images of the continuum, the integrated $\mathrm{H} \alpha$ brightness and the radial velocity distribution.

\subsection{Cleaning the observed velocity field}

The velocity in a given pixel is determined $\pm k \times F S R$, where $k$ is the maximum number of interference orders which can be seen. It depends on the width of the interference filter used during the observations ( $k=1$ here). As the $\mathrm{H} \alpha$ velocity range measured over the entire galaxy exceeds the FSR $\left(\sim 450 \mathrm{~km} \mathrm{~s}^{-1}\right.$ compared to the $F S R$ of $378 \mathrm{~km} \mathrm{~s}^{-1}$ ), radial velocities outside the FSR are shifted by $\pm F S R$. To correct for this effect, the FSR value was added to, or subtracted from, the radial velocity in each pixel affected by this bias.

The continuum level in the vicinity of the $\mathrm{H} \alpha$ line is very strong in the central region of the galaxy, where the signal from the bar and from the nucleus exceeds by a factor of more than 10 the continuum level of most of the HII regions in the disc (see Fig. 1b). In this region, the $\mathrm{H} \alpha$ emission lines are noisy and their width could exceed the FSR. Moreover, line-ofsight velocity gradients, like those measured in the central part of the molecular bar by Thornley et al. (1999), are larger than the FSR and prevent the determinination of the exact position of the line barycentres. We therefore excluded from the velocity field those pixels in which it was impossible to properly extract the emission line and to derive a radial velocity. This leads to an absence of information on the kinematics of the innermost region, mainly for deprojected radii $\lesssim 8^{\prime \prime}$ along the major axis.

\section{Distribution of the ionized gas}

\subsection{The continuum emission}

The continuum in the vicinity of the $\mathrm{H} \alpha$ line (Fig. 1b) is mainly concentrated in the central region of the galaxy, and is fainter in the bar and in the spiral structure. It peaks in the nucleus, which highest intensity is located at RA $=11^{\mathrm{h}} 20^{\mathrm{m}} 15^{\mathrm{s}} .05$, Dec $=+12^{\circ} 59^{\prime} 29^{\prime \prime} .7$ (J2000). Two other fainter peaks of the continuum are associated with very bright HII regions at the starting points of the spiral arms (see Figs. 1c, d and 2), probably marking the outermost limits of the bar.

The continuum signal follows the stellar bar, for which we can estimate a position angle of $155 \pm 5^{\circ}$ from the orientation of the line connecting the two continuum peaks near its opposite ends (see the dashed line in Fig. 1b). This agrees well with the position angle of $\sim 157^{\circ}$ derived from isophotal ellipses fitted to a $K$-band image by Regan \& Elmegreen (1997). Our position angle of the bar is also in agreement (within the errors) with that found for the molecular bar observed in CO by Zhang et al. (1993). Twists of the continuum isophotes in the nuclear region visible in our data are probably due to projection effects and to important dust extinction in the bulge (Smith et al. 1994), rather than to a genuine nuclear triaxial structure. Moreover no isophotal twists in this region were observed in the above mentioned $K$-band image (Regan \& Elmegreen 1997).

\subsection{The integrated $\mathrm{H} \alpha$ map}

Our map of the $\mathrm{H} \alpha$ emission integrated over the velocity channels where line emission is seen (Figs. 1c, $d$ and 2 for the inner regions) is consistent with the $\mathrm{H} \alpha+[\mathrm{NII}]$ image by Smith et al. (1994), except for the nuclear region where our map does not show the $\mathrm{H} \alpha$ emission (see Sect. 2.3). The brightest HII regions visible in our map are located in the spiral arms, at both ends of the bar.

In Fig. 1c, we see that the distribution of the ionized gas follows the CO distribution from Regan et al. (1999). Within the two spiral arms and near the ends of the bar, where two strong "twin peaks" are found both in the $\mathrm{H} \alpha$ and CO lines, massive star formation occurs in regions which seem depleted in CO. Offsets of a few arcsecs are found between $\mathrm{H} \alpha$ and $\mathrm{CO}$ maxima locations.

Another difference can be seen at the bar location. If we do not take into account the strong central CO condensation, where no comparison can be made with our $\mathrm{H} \alpha$ map, the $\mathrm{CO}$ data show emission along the leading sides of the stellar bar, while few faint HII regions (labelled "a", "b", "c", "d" and "e" in Figs. 1c and 2) are seen at the external side of the CO S-shape, as mentioned by Sheth et al. (2002). The small number of $\mathrm{H} \alpha$ regions that are seen along the bar is consistent with what is observed in early-type barred spirals (Phillips 1996). East and West of the bar, we notice $\mathrm{H} \alpha$ arclets traced by faint HII regions (labelled "f", "g", "h", “i”, “j”, "k" and "l”) amidst a more diffuse ionized emission.

A bright HII knot (labelled "m") and a structure which forms a segment (labelled "no") roughly parallel to the western arm are seen in the interarm region in the South. The northernmost end of the eastern arm splits into two armlets, as well as the dust lanes in this area in the broad-band optical image (Fig. 1a).

\section{Kinematics of the ionized gas}

\subsection{The velocity field}

Figure 1d shows the isovelocity contours of ionized gas in NGC 3627 obtained after a spatial smoothing of the fullresolution data cube using a 2D Gaussian with a $F W H M$ of $3 \times$ 3 pixels, superimposed on the integrated $\mathrm{H} \alpha$ map smoothed in the same way. The velocity field shows a considerable asymmetry with respect to the major axis. At large radii the isovelocity lines are more strongly twisted in the South than in the North. At smaller radii we notice strong velocity gradients which are roughly parallel to the slightly curved parts of the CO bar and coincide with some dust lanes (see e.g. near the "i", "j", "k" and "l" locations). 

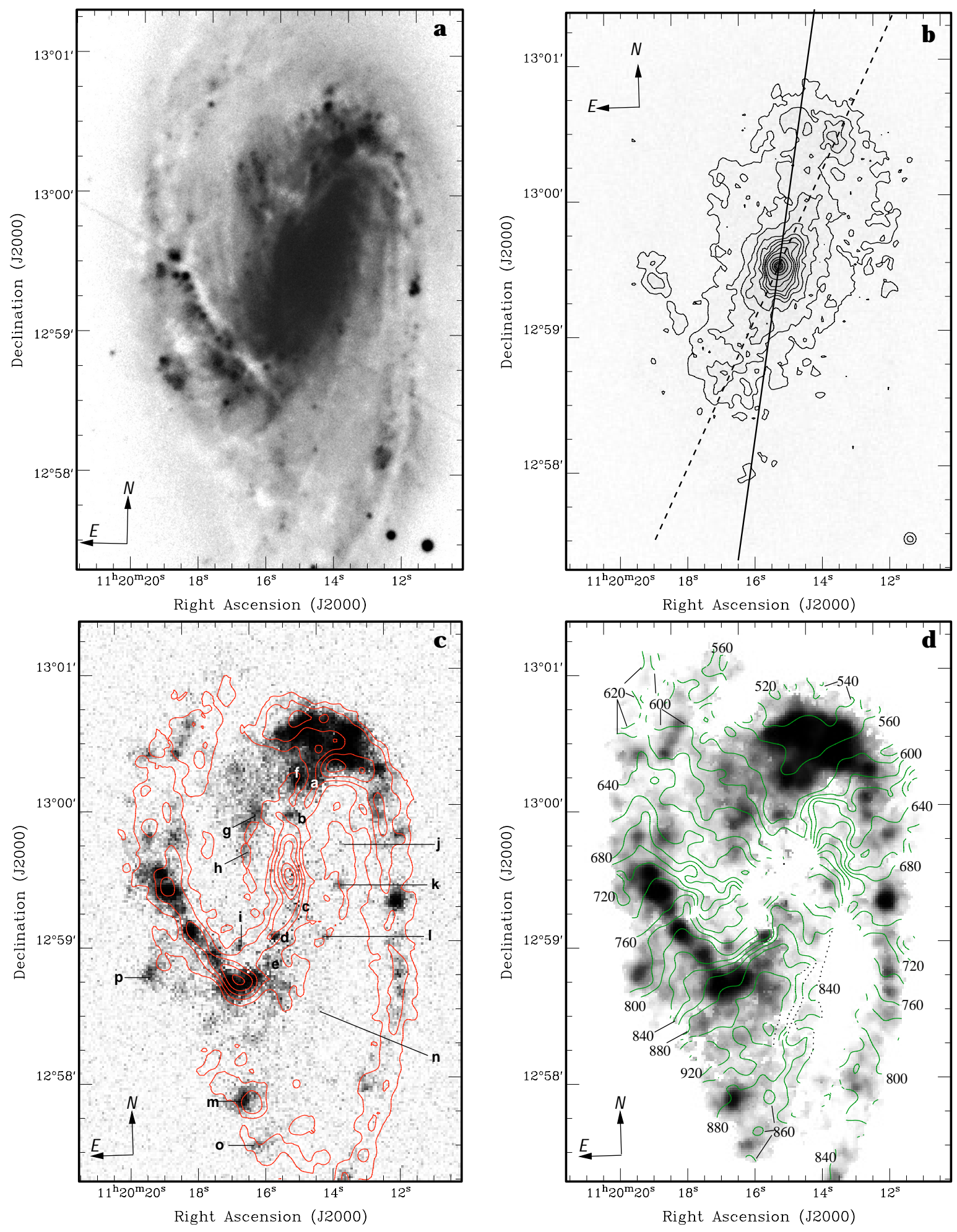

Fig. 1. a) Optical broad-band image of NGC 3627, taken from the Anglo Australian Observatory's website. b) Continuum map of NGC 3627 from our observations. The contour levels are at $(3,5,7,9,11,13,15,17,21,31,42,52,57) \times \sigma_{\text {cont }}$ above the mean continuum background level ( $\sigma_{\text {cont }}$ represents the dispersion around this mean level in arbitrary units). The solid line indicates the direction of the kinematical major axis of the galaxy and the dashed line indicates the direction of the major axis of the optical bar. c) Grey scale image (full resolution) of the $\mathrm{H} \alpha$ integrated emission line of NGC 3627 with superimposed $\mathrm{CO}(1-0)$ intensity contours (Regan et al. 1999) with levels of (4, 12, 26, 50, $90,130,170) \mathrm{Jy} / \mathrm{beam} \times \mathrm{km} \mathrm{s}^{-1}$. The letters indicate the positions of HII regions along the leading sides of the bar, in the H $\alpha$ arclets and in the interstellar medium (see text for details). d) Isovelocity contours of our $\mathrm{H} \alpha$ velocity field superimposed on a smoothed (by $3 \times 3$ pixels $F W H M)$ greyscale of our $\mathrm{H} \alpha$ integrated brightness. The contour levels are separated by $20 \mathrm{~km} \mathrm{~s}^{-1}$. 


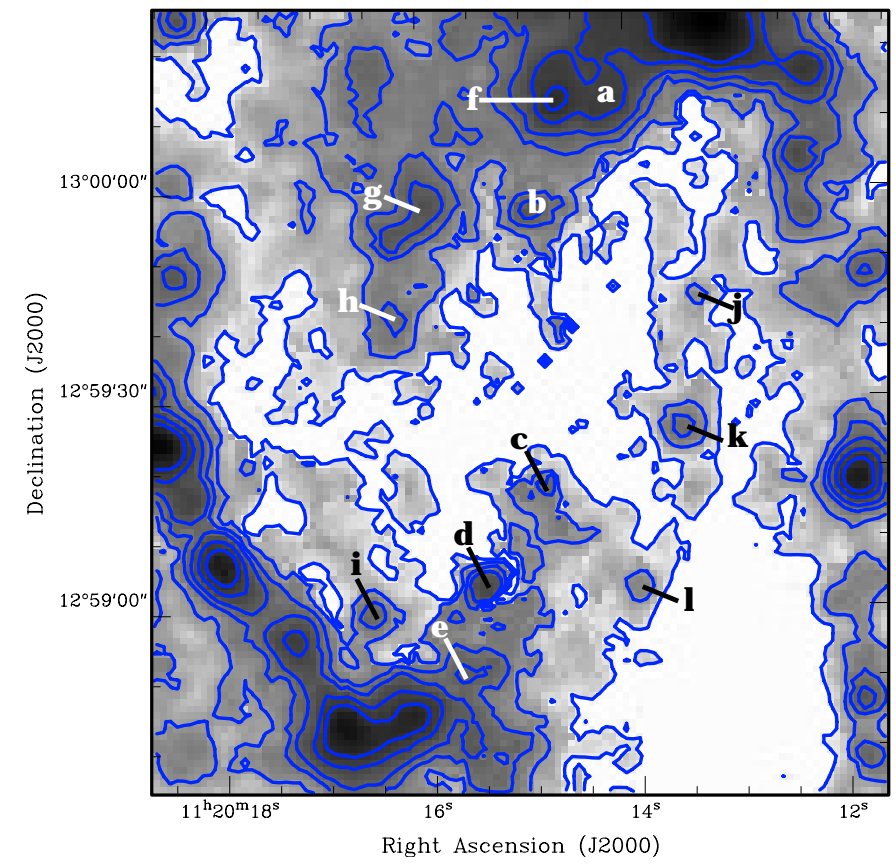

Fig. 2. Enlarged $\mathrm{H} \alpha$ image of the inner regions of NGC 3627 from Fig. 1d. Contours of the smoothed $\mathrm{H} \alpha$ map are at confidence levels of $(3,10.5,22,37,57,76) \times \sigma_{\text {mono }}$ above the mean monochromatic background level $\left(\sigma_{\text {mono }}\right.$ represents the dispersion around this mean level in arbitrary units) and superimposed on a grey-scale representation of the same $\mathrm{H} \alpha$ image. The letters indicate the positions of some HII regions along the leading sides of the bar and in the $\mathrm{H} \alpha$ arclets (see text for details).

In the eastern arm, broad and smooth wiggles are present along the brightest HII regions. They become narrower and double as the arm bifurcates into two armlets towards the North-East. We notice that the clump labelled "p" (RA = $11^{\mathrm{h}} 20^{\mathrm{m}} 19^{\mathrm{s}} .20$, Dec $\left.=+12^{\circ} 58^{\prime} 47^{\prime \prime} .0\right)$ has its radial velocity $\left(\sim 760 \mathrm{~km} \mathrm{~s}^{-1}\right)$ about $60 \mathrm{~km} \mathrm{~s}^{-1}$ lower than nearby velocities in the arm at the same azimuthal angle. Perturbed isovelocity contours are also present along the western arm and probably reveal gas streaming motions.

\section{2. $\mathrm{H} \alpha$ rotation curve}

We first fitted the systemic velocity $\left(V_{\text {sys }}\right)$, the inclination $(i)$, the position angle (PA) and the kinematical centre in such a way that the approaching and receding parts of the rotation curve are as symmetric as possible, i.e. by minimizing the differences between the rotation speeds for these disc halves. Only points within a sector of $\pm 45^{\circ}$ around the major axis were used. This resulted in a systemic velocity of $727 \pm 5 \mathrm{~km} \mathrm{~s}^{-1}$, a kinematical inclination of $65 \pm 5^{\circ}$ and a position angle of the kinematical major axis of $170^{\circ} \pm 5^{\circ}$. The kinematical centre coordinates correspond to those of the peak in our optical continuum map (see Sect. 3.1).

In a second step, we used the rotcur task of the GIPS Y data reduction package (Begeman 1989) with the derived kinematical parameters. The resulting rotation curves for the approaching and receding sides are shown in Fig. 3a. In the most symmetric part of the curves $\left(35^{\prime \prime}<R<70^{\prime \prime}\right)$, the differences

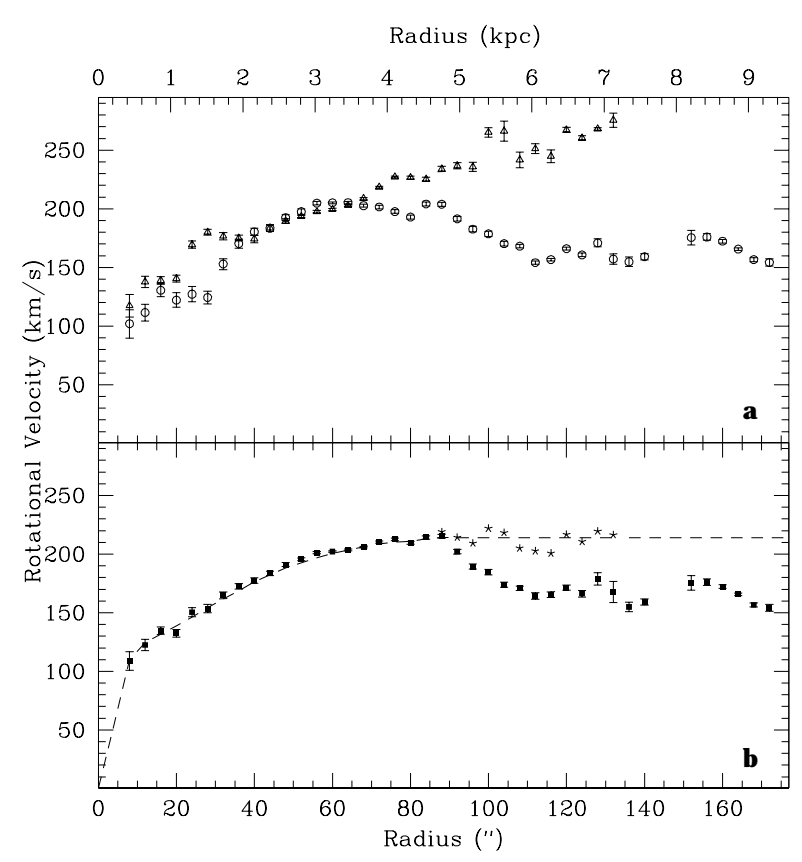

Fig. 3. $\mathrm{H} \alpha$ rotation curve of NGC 3627. a) The rotation curves of the approaching and the receding sides fitted separately: open circles and triangles are for the receding and approaching sides respectively. b) The global rotation curve, fitted to both sides of the disc (squares). Stars indicate the average of the receding and the approaching sides curves at radii above $88^{\prime \prime}$. The dashed line shows the adopted best model for the global rotation curve.

between the velocities of the two sides are small $\left(\sim 4 \mathrm{~km} \mathrm{~s}^{-1}\right.$ in average) as well as the formal error-bars $\left(\sim 3 \mathrm{~km} \mathrm{~s}^{-1}\right.$ in average).

Figure 3 a shows that an asymmetry occurs in the inner disc between radii of $25^{\prime \prime}$ and $34^{\prime \prime}$, with a maximum difference of $\sim 60 \mathrm{~km} \mathrm{~s}^{-1}$ at a radius $R=28^{\prime \prime}$. This discrepancy is due to the bar which induces non-circular gas motions in the innermost part of the disc. Non-circular motions also probably occur at radii between $70^{\prime \prime}$ and $85^{\prime \prime}$, where the two halves curves show a smaller separation $\left(<35 \mathrm{~km} \mathrm{~s}^{-1}\right)$. The most significant asymmetry is observed at the largest radii $\left(R>88^{\prime \prime}\right)$. For the receding southern half of the disc, the decreasing rotational velocities are due to the southernmost regions in the galactic disc located at radii between 88" and 140" (regions "m" and "o" plus the tip of the western spiral arm in Figs. 1c, d). For $R>150$ " they are due to the southern tip of the western spiral arm. For the approaching half the rotational velocities are increasing with radius, which is mainly due to the northern tip of the eastern arm at $R>100^{\prime \prime}$.

In Fig. 3b, for radii beyond $88^{\prime \prime}$ the global rotation curve (derived for both disc sides simultaneously, see Table 3) shows a declining trend because it is weighted by more pixels on the southern side of the velocity field than on the northern side. By contrast, the average observed rotation curve, which was obtained by applying a uniform weighting for the two sides, presents small ripples around a value of $\sim 210 \mathrm{~km} \mathrm{~s}^{-1}$, but globally follows a flat trend. 


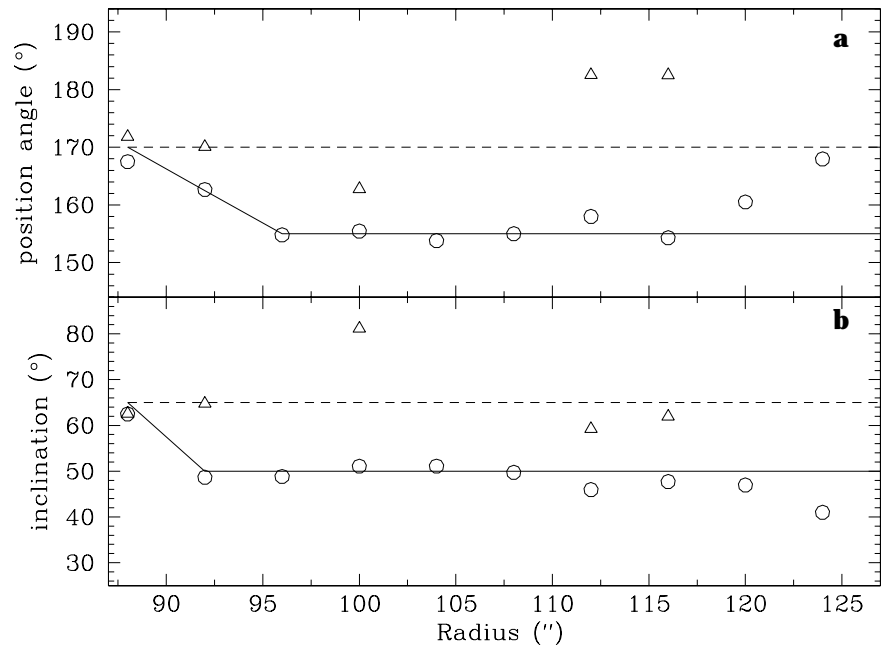

Fig. 4. Position angle a) and inclination b) as function of deprojected galactocentric radius, starting from $R=88^{\prime \prime}$. Open circles and the solid line represent the fitted points and the adopted model parameters for the receding side, while open triangles and the dotted line show the same quantities for the approaching side.

\subsection{Tilted-ring model}

In order to understand why the observed rotation curves on Fig. 3a do not show a classical plateau beyond $88^{\prime \prime}$, we analyzed in detail the separate model fits for the approaching and receding sides with rotcur. The systemic velocity and the kinematical centre position were kept fixed during the fitting procedure. We also fixed the maximal rotational velocity at a value of $\sim 210 \mathrm{~km} \mathrm{~s}^{-1}$, as can be inferred from the observed average rotation curve (see Sect. 4.2).

Figure 4 presents the results of the fits. Only points with reliable and reasonable values having an error-bar $\leq 5^{\circ}$, are shown. Some values at radii between $96^{\prime \prime}$ and $112^{\prime \prime}$ are therefore not taken into account for the approaching side and at radii $R \geq 124^{\prime \prime}$ for the receding side.

The figure shows that, though the scatter seems high at the largest radii on the receding side, both the derived inclinations and position angles lie $\sim 15^{\circ}$ below the values found for smaller radii. This indicates a warped outer disc on the southern side of NGC 3627. For the approaching side, only few points remain. They show that the inclination is in relative good agreement with the inner disc value of $65^{\circ}$, except at $R=100^{\prime \prime}$. For $R<100^{\prime \prime}$, the fitted position angle is very close to the PA of $170^{\circ}$ in the inner disc and for $R \geq 112^{\prime \prime}$ it is $\sim 15^{\circ}$ higher than $170^{\circ}$. Clearly, the two sides of NGC 3627 do not exhibit the same trend for PA and $i$ variations, contrary to what is usually observed for a "classical" symmetrical warp.

A detailed analysis of the errors of $i$ and PA for the approaching side shows that they are at least two times larger than those found for the receding half. Moreover, points outside $R=100^{\prime \prime}$ are due to a few pixels at the northern tip of the eastern spiral arm only; the uncertainties are too important here and we will not take into account these fitted values at radii exceeding $88^{\prime \prime}$ for the approaching side.
Table 3. H $\alpha$ rotation curve of NGC 3627 at 4 " binning from rotcur.

\begin{tabular}{cccc}
\hline \hline$R(\operatorname{arcsec})$ & $V_{\text {rot }}\left(\mathrm{km} \mathrm{s}^{-1}\right)$ & $R(\operatorname{arcsec})$ & $V_{\text {rot }}\left(\mathrm{km} \mathrm{s}^{-1}\right)$ \\
\hline 8 & $109 \pm 8$ & 48 & $191 \pm 2$ \\
12 & $123 \pm 5$ & 52 & $196 \pm 1$ \\
16 & $135 \pm 3$ & 56 & $201 \pm 1$ \\
20 & $132 \pm 3$ & 60 & $202 \pm 1$ \\
24 & $150 \pm 4$ & 64 & $204 \pm 1$ \\
28 & $154 \pm 4$ & 68 & $206 \pm 1$ \\
32 & $165 \pm 3$ & 72 & $210 \pm 1$ \\
36 & $173 \pm 2$ & 76 & $213 \pm 1$ \\
40 & $177 \pm 2$ & 80 & $210 \pm 1$ \\
44 & $184 \pm 2$ & 84 & $214 \pm 1$ \\
\hline
\end{tabular}

\subsection{Residual velocity field}

We constructed a model velocity field (Fig. 5a) and a residual (observation minus model) velocity map (Fig. 5b). We used the flat model rotation curve (dashed line in Fig. 3b, see also Table 3). Inside $R=88^{\prime \prime}$, we used the constant $i$ and PA derived from fits to the inner disc $\left(65^{\circ}\right.$ and $170^{\circ}$, respectively) for both sides, whereas beyond $88^{\prime \prime}$, the full and dotted lines in Fig. 4 indicate the values we used as model parameters for the receding and approaching halves, respectively. A practical difficulty in this model is to ensure the smooth joining of the two velocity fields at large radii along the minor axis $\left(\mathrm{PA}=80^{\circ}\right)$, which mainly comes down to masking selected points in the image.

Except for small localized regions, the smallest residuals (between -15 and $+15 \mathrm{~km} \mathrm{~s}^{-1}$ ) are found in bright HII clumps in spiral arms, in the South ("m" and "o" regions) and at the end of the western arm. Low residuals indicate that very few streaming motions or radial inflow or outflow occur where the spiral arms begin.

We notice high "symmetric" residuals (in absolute values) west and east of the bar and inside the $\mathrm{H} \alpha$ arclets. Here, the highest residuals are located near the minor axis (up to $+65 \mathrm{~km} \mathrm{~s}^{-1}$ on its western side and $-75 \mathrm{~km} \mathrm{~s}^{-1}$ on its eastern side).

In the western arm, outside $88^{\prime \prime}$ (North of the minor axis), residual velocities are between -35 and $-15 \mathrm{~km} \mathrm{~s}^{-1}$. If we consider that the galaxy has trailing arms and that the eastern half is the closest to the observer, this region is on the far side and its negative residuals could correspond to radial inflow streaming motions.

Asymmetries were observed in the southern half of the $\mathrm{CO}$ velocity field (Reuter et al. 1996) and Zhang et al. (1993) also showed a perturbed HI velocity field in the South, pointing out on a non-corotating region in the South-East of the disc. The "m" H $\alpha$ clump corresponds in position and in velocity to the HI "peculiar velocity clump" observed by Zhang et al. (1993). They proposed that this clump is situated at a high latitude, outside the plane of the disc. The residual velocity field shows that it is actually in the warped southern part of the disc, like its surroundings, and we do not observe any peculiar velocities at this location. Moreover, the tip of the western arm also forms part of the warp. The HI velocity field of Zhang et al. (1993), which has a better spatial coverage but a considerably lower 

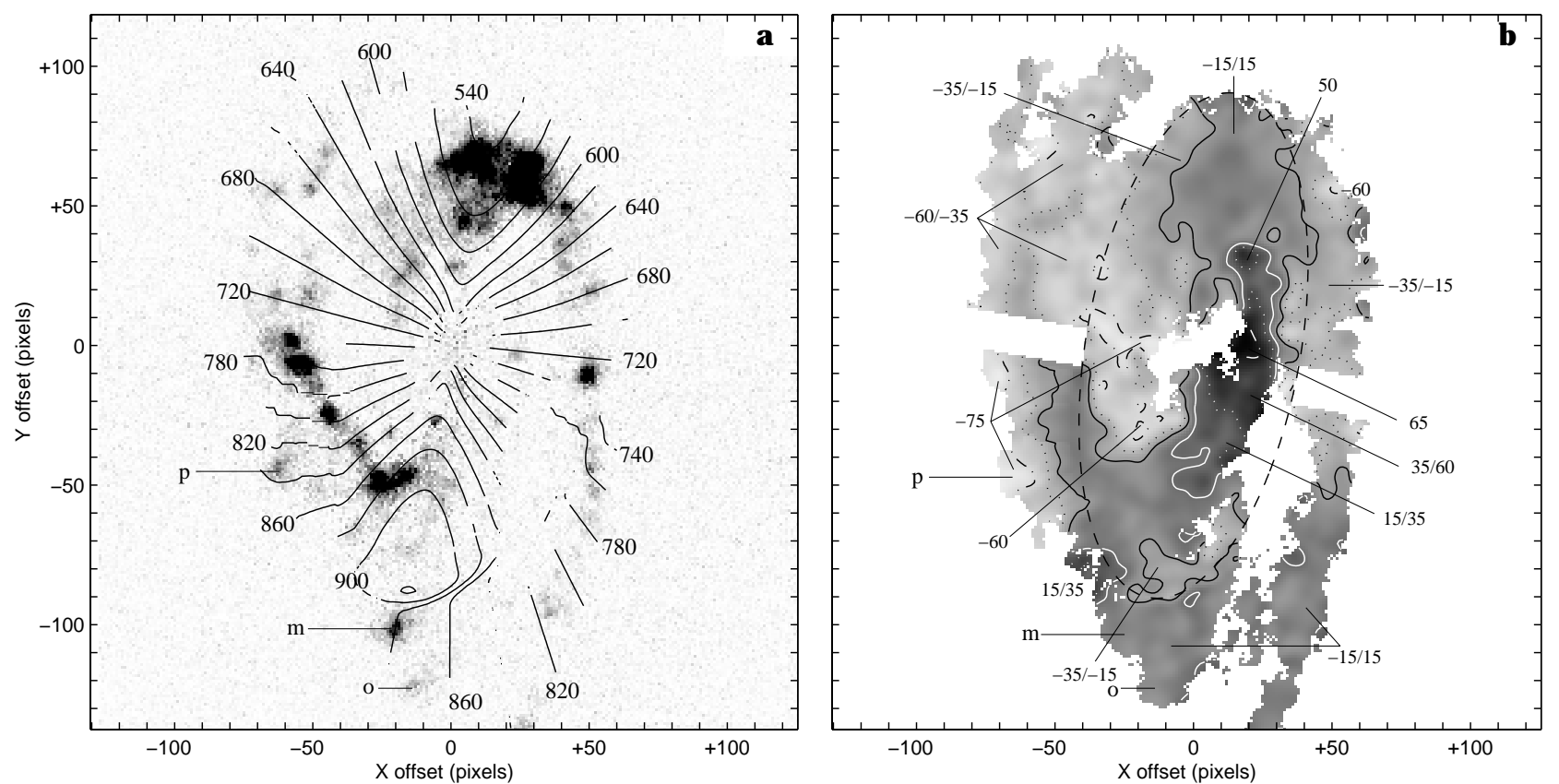

Fig. 5. a) Model velocity field superimposed on our high-resolution $\mathrm{H} \alpha$ image. Velocity contours are separated by $20 \mathrm{~km} \mathrm{~s}^{-1}$. b) Grey-scale representation of the residual velocity field (observation minus model). The dashed ellipse represents the $R=88^{\prime \prime}$ limit between the axisymmetric model and the titled-ring outer disc models. In each image, the $[0,0]$ point refers to the kinematical centre and the letters " $m$ ", "o" and "p" to HII regions discussed in the text.

spatial resolution $\left(30^{\prime \prime}\right)$ than our data, shows a clear indication of a warped outer disc, like our data. However, no model fit was made to this velocity field, so no comparison is possible.

Finally, extremely high residual velocities occur in the "p" region (up to $\sim-75 \mathrm{~km} \mathrm{~s}^{-1}$ ). This value is consistent with the difference of $60 \mathrm{~km} \mathrm{~s}^{-1}$ observed in the velocity field between this clump and its surroundings (see Sect. 4.1). We may suppose that it lies currently off the galactic plane. It could be located under the galactic plane with a $z$-velocity component which tends to bring back the region towards the disc. On the other hand, if it is located into the galactic plane it would have a large radial outflow velocity component. The optical morphology of the end of the eastern arm shows evidence for structures lifted above the principal plane of the disc. The high residual velocities $\left(<-35 \mathrm{~km} \mathrm{~s}^{-1}\right)$ observed here illustrate the difficulty to model the kinematics of this perturbed arm with our data only.

\section{Dynamical analysis}

\subsection{The $\mathrm{H} \alpha_{\alpha}$ inner ring}

As shown in Figs. 1c, 2, it is seen that $\mathrm{CO}$ arclets, which were interpreted as parts of an inner molecular ring by Regan et al. (2002), superimpose well onto the $\mathrm{H} \alpha$ arclets, namely the "fgh" and "jkl" segments. Here, our observed velocity field, traced by the diffuse ionized gas and faint HII regions, and our residual velocity field clearly show the signature of deviations from pure circular motions. Moreover, our velocity field looks similar to the one seen in the inner ring model of Regan et al. (2002), though only a qualitative comparison is possible since the authors do not give velocity contour values in their Fig. 6. They derived a velocity field from the standard hydrodynamical model simulations of Piner et al. (1995). As in Regan et al. (2002), we find that ionized gas in the arclet west of the bar is more red-shifted than inside the western arm, and symmetrically in the eastern part, velocity components are blue-shifted. Hence, both the morphology and kinematics of the $\mathrm{H} \alpha$ and $\mathrm{CO}$ are consistent with each other. We deduce from our data that this inner ring lies between deprojected radii of $\sim 30^{\prime \prime}$ to $\sim 60^{\prime \prime}$ with maximum intensities at $R \simeq 50^{\prime \prime}$ (see e.g. the region labelled $k$ at $\mathrm{RA}=11^{\mathrm{h}} 20^{\mathrm{m}} 13 \mathrm{~s} .50$, Dec $=+12^{\circ} 59^{\prime} 26^{\prime \prime} .3$ ). The major axis position angle of the annular structure is not easy to determine and we can only restrict it between the mean major axis PA of the bar $\left(155^{\circ}\right)$ and the overall major axis PA of the galaxy $\left(170^{\circ}\right)$.

\subsection{Position of the corotation radius}

Resonances in barred galaxies are related to the pattern speed of the stellar bar, $\Omega_{\mathrm{p}}$, which can be determined at the corotation (CR) radius. In early-type barred spirals, $\mathrm{CR}$ is located near the end of the bar, between $R=R_{\mathrm{bar}}$ and $R=1.4 R_{\mathrm{bar}}$ (Elmegreen 1996; Elmegreen et al. 1996).

Das et al. (2003) give a bar semi-major axis of 49" for a galaxy inclination of $63^{\circ}$, which is in very good agreement with our estimate of $65^{\circ}$ taking into account the error-bars. We thus expect a corotation radius $R_{\mathrm{c}}$ between $49^{\prime \prime}$ and $69^{\prime \prime}$, i.e. between 2.6 and $3.7 \mathrm{kpc}$ for the assumed distance of $11.1 \mathrm{Mpc}$ (Saha et al. 1999).

This result differs from those found by Zhang et al. (1993) and Reuter et al. (1996). Our H $\alpha$ curve is hardly comparable to the $\mathrm{HI}$ and $\mathrm{CO}$ curves, partly due to their poorer angular 


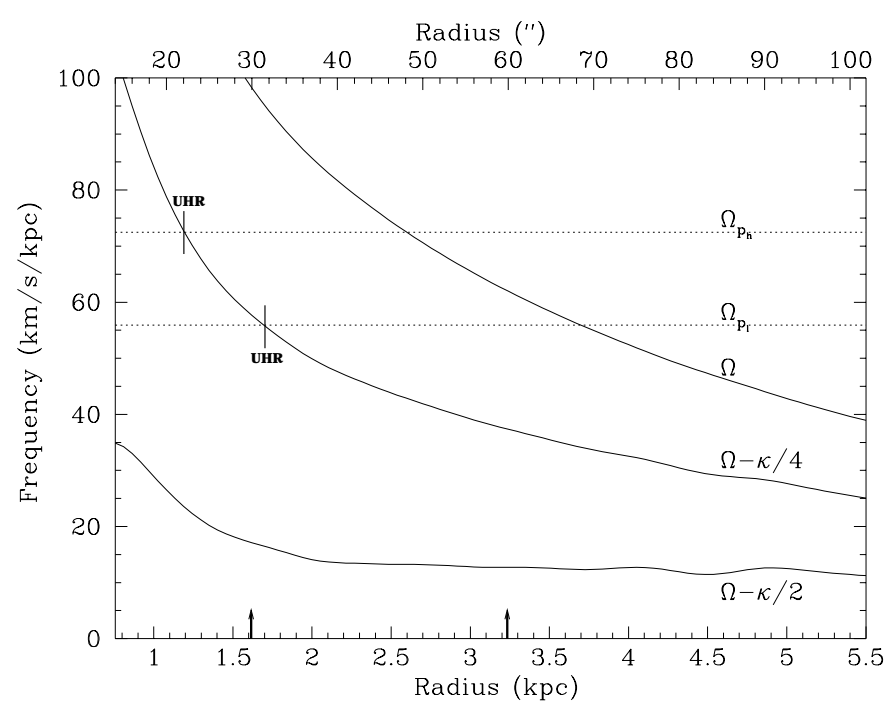

Fig. 6. Angular velocities in NGC 3627 as function of radius. The dotted horizontal lines indicate a bar pattern speed of, respectively, $56 \mathrm{~km} \mathrm{~s}^{-1} \mathrm{kpc}^{-1}\left(\Omega_{\mathrm{p}_{1}}\right)$ and $72 \mathrm{~km} \mathrm{~s}^{-1} \mathrm{kpc}^{-1}\left(\Omega_{\mathrm{p}_{\mathrm{h}}}\right)$, giving an ultraharmonic resonance between 1.2 and $1.7 \mathrm{kpc}$ radius, with the $\Omega-\kappa / 4$ curve. The arrows indicate the extension of the annular structure in the galaxy, between $30^{\prime \prime}$ and $60^{\prime \prime}$.

resolution, $30^{\prime \prime}$ for the $\mathrm{HI}$ data and $12^{\prime \prime}$ for the $\mathrm{CO}$ data (respectively). Since in the inner part of NGC 3627 the CO emission is dominated by the bar, discrepancies with our rotation curve could be explained by the fact that non-circular orbits affect more strongly the $\mathrm{CO}$ velocity field.

We applied the "geometric phase" method (Canzian 1993) in order to test whether our CR position is valid or not. The test is based on a morphological analysis of a residual velocity field and shows that inside CR one pair of approaching (negative residuals) and receding (positive residuals) spiral arm structures are seen, while outside CR there are three pairs of approaching-receding spiral arms. An example can be seen in Sempere et al. (1995), who applied the test to the galaxy NGC 4321. They showed an extended region where the change between one pair to three pairs of spiral arms occurs. For NGC 3627, we subtracted from our $\mathrm{H} \alpha$ velocity field a simple model based on the adopted rotation curve (Fig. 3b) and $i=65^{\circ}$ and $\mathrm{PA}=170^{\circ}$ throughout the whole disc. Although one of the receding spirals of the $m=3$ mode is hardly detectable, we can clearly define a transition region, where a $m=1$ spiral pattern develops to the $m=3$ mode, between $\sim 60^{\prime \prime}$ and $\sim 80^{\prime \prime}$ (as it is also seen in Fig 5b).

Zhang et al. (1993) and Reuter et al. (1996) both fixed CR at a seemingly much too large radius $\left(R=3{ }^{\prime} 6\right)$, i.e. nearly three times more than our upper limit of $69^{\prime \prime}$. This result cannot be due to the inclination difference of $5^{\circ}$ between the $\mathrm{H} \alpha$ and the two other studies ( $i=60^{\circ}$ in Zhang et al. and Reuter et al.). In Reuter et al. (1996), there seems to be confusion between radii and diameters of structures, leading to a flawed analysis. We will thus limit CR in NGC 3627 between our estimates of $R=$ $49^{\prime \prime}$ and $R=69^{\prime \prime}$.

\subsection{The pattern speed of the bar and the resonances}

We clearly see in the $\mathrm{H} \alpha$ maps (Figs. 1c, d and 2) that the most intense and extended emissions in the arclets are located to the west and east of the leading sides of the bar (see the "fgh" segment and the " $k$ " peak). On the other hand, emissions near the trailing sides of the bar are weaker. In her models, Athanassoula (1992) found stronger gas density enhancements on the leading sides of a bar than on its trailing sides. These enhancements are tightly related to the periodic orbits around and inside the 4:1 resonance.

To estimate resonances in NGC 3627, we first determined the angular velocities $\Omega$ and $\Omega-\kappa / m$, where $\kappa$ is the epicyclic frequency and " $\mathrm{m}$ " an integer. We assume that at any radius the gas dynamics is governed by the adopted model rotation curve (see Fig. 3b), and that the epicycle approximation is valid.

Figure 6 shows that the deduced values of $\Omega_{p}$ lie between 56 and $72 \mathrm{~km} \mathrm{~s}^{-1} \mathrm{kpc}^{-1}$. This result is in good agreement with the recent estimate of $55 \mathrm{~km} \mathrm{~s}^{-1} \mathrm{kpc}^{-1}$ by Sheth et al. (2002).

We find the position of the ultra-harmonic resonance (UHR) where $\Omega_{\mathrm{p}}=\Omega-\kappa / 4$, at $1.2 \lesssim R \lesssim 1.7 \mathrm{kpc}\left(22^{\prime \prime} \lesssim\right.$ $\left.R \lesssim 32^{\prime \prime}\right)$. It is important to note that the location of the UHR at $32^{\prime \prime}$ coincides with the arc-like structures both seen in the $\mathrm{H} \alpha$ and $\mathrm{CO}$ maps. It is therefore possible that the $\mathrm{H} \alpha$ arclets are due to ultra-harmonic resonant orbits in the galaxy. This would be consistent with what is generally observed in barred galaxies having an inner ring (Buta \& Combes 1996). As a consequence, the CR radius is rather located at about 70", corresponding to the highest "allowed" value at $R=1.4 R_{\mathrm{bar}}$ and the bar has an angular velocity of $56 \mathrm{~km} \mathrm{~s}^{-1} \mathrm{kpc}^{-1}$.

Regan et al. (1999) argued for a nuclear ring in NGC 3627, though without being able to locate its edges. Unfortunately, our lack of $\mathrm{H} \alpha$ data in the nuclear region does not allow any identification of its signature.

\section{Concluding remarks}

In this paper we have studied the $\mathrm{H} \alpha$ distribution and kinematics of the Sb weakly-barred spiral galaxy NGC 3627. In the inner disc, our high angular resolution monochromatic $\mathrm{H} \alpha$ map is dominated by HII regions along the leading sides of the bar and by a weak and diffuse ring, while at larger galactocentric radii, bright HII regions are present in the spiral arms as well as in the arm-interarm medium.

As especially the velocity field of the receding side shows signs of a kinematical warp in its outer regions, we fitted a tilted-ring model to it, confirming the warped nature of the disc, which was found to be inclined by about $15^{\circ}$ with respect to the plane of the inner disc. This model uses a flat outer rotation curve which reaches its plateau of $\sim 210 \mathrm{~km} \mathrm{~s}^{-1}$ at a radius of $\sim 90^{\prime \prime}$.

In the $\mathrm{H} \alpha$ map and in the velocity field, the signature of a ring-like structure is evident between $30^{\prime \prime}$ and $60^{\prime \prime}$. Although a hydrodynamical model of this ring (Regan et al. 2002), based on recent $\mathrm{CO}$ observations, resulted in a velocity field that resembles ours, only a qualitative comparison could be made. We have shown that the ring is likely due to ultra-harmonic 
resonant orbits, choosing the corotation location outside the end of the bar, at $\sim 70^{\prime \prime}$ radius. The bar rotates fast, with a frequency of $56 \mathrm{~km} \mathrm{~s}^{-1} \mathrm{kpc}^{-1}$.

Rings in barred galaxies, principally early-type ones, could be formed by gas accumulation at resonances (Buta \& Combes 1996). Strong non-circular motions can occur in one ring where elliptical orbits can better mimic the observed gas kinematics than a pure circular rotation pattern (Sakamoto et al. 2000). Actually, there exist few cases of ringed barred galaxies that have been the subject of a complete dynamical analysis (see e.g. Salo et al. 1999; Buta et al. 2001). NGC 3627 is similar to other galaxies having an inner ring around a bar, roughly sharing the same orientation as the bar major-axis, and with strong non-circular motions in its ring. The formation of the inner ring can then be related to the presence of the bar which nevertheless has a larger amount of molecular gas (Regan et al. 2002). Regan et al. also noticed that the inner ring is not prominent in their $K$-band image, indicating a young age of its stellar population. The presence of an inner ring in NGC 3627 could be a transient phenomemon.

Warps are common in disc galaxies and are more often seen in HI observations than in optical ones. Warps are more frequent and larger among discs within rich environments than among isolated galaxies (Reshetnikov \& Combes 1998; García-Ruiz et al. 2002). Tidal effects indeed play an important role in triggering a disc warping though other mechanisms, like a secondary gas infall, a magnetic field or a misalignment of the disc and dark halo angular momenta, could excite or maintain warps (see García-Ruiz et al. 2002 and references therein). In NGC 3627, the optical warp is large and is only seen in the southern side of the galaxy. Like for the other perturbations observed in the Leo Triplet, the plume between NGC 3627 and NGC 3628, the giant extension from NGC 3628 to the East and the disturbed outskirts of the NGC 3627 disc both detected in HI observations (Haynes et al. 1979), the tidal interaction between the two largest members of the Leo Triplet is likely at the origin of the optical warping observed in NGC 3627.

Acknowledgements. We want to thank David Malin for providing us with the image of NGC 3627 (see http://www.aao.gov.au/ images for more information). We thank Marek Urbanik for suggesting the observation of this object and for helpful discussion, and Olivia Garrido for her help during the observation at OHP. We have made use of the LEDA database (http: //leda.univ-lyon1. fr).

\section{References}

Amram, P., Le Coarer, E., Marcelin, M., et al. 1992, A\&AS, 94, 175 Athanassoula, E. 1992, A\&A, 259, 345

Begeman, K. G. 1989, A\&A, 223, 47

Buta, R., \& Combes, F. 1996, Fund. Cosmic Physics, 17, 95

Buta, R., Ryder, S. D., Madsen, G. J., et al. 2001, AJ, 121, 225

Canzian, B. 1993, ApJ, 414, 487

Das, M., Teuben, P. J., Vogel, S. N., et al. 2003, ApJ, 582, 190

de Vaucouleurs, G., de Vaucouleurs, A., Corwin, H. G. Jr., et al. 1991, Third Reference Catalogue of Bright Galaxies (New York: Springer-Verlag)

Elmegreen, B. G. 1996, in Barred Galaxies, IAU Coll. 157, ed. R. Buta, B. Elmegreen, \& D. Crocker, ASP Conf. Ser., 91, 197

Elmegreen, B. G., Elmegreen, D. M., Chromey, F. R., et al. 1996, AJ, 111,2233

García-Ruiz, I., Sancisi, R., \& Kuijken, K. 2002, A\&A, 394, 769

Garrido, O., Marcelin, M., Amram, P., et al. 2002, A\&A, 387, 821

Garrido, O., Marcelin, M., Amram, P., et al. 2003, A\&A, 399, 51

Haynes, M. P., Giovanelli, R., \& Roberts, M. S. 1979, ApJ, 229, 83

Ho, L. C., Filippenko, A. V., \& Sargent, W. L. W. 1997, ApJS, 112, 315

Marcelin, M., Lecoarer, E., Boulesteix, J., et al. 1987, A\&A, 179, 101

Otmianowska-Mazur, K., Elstner, E., Soida, M., et al. 2002, A\&A, 384,48

Paturel G., Fouqué P., Buta, R., et al. 1991, A\&A, 243, 319

Phillips, A. C. 1996, in Barred Galaxies, IAU Coll. 157, ed. R. Buta, B. Elmegreen, \& D. Crocker, ASP Conf. Ser., 91, 44

Piner, B. G., Stone, J. M., \& Teuben, P. J. 1995, ApJ, 449, 508

Regan, M., \& Elmegreen, D. M. 1997, AJ, 114, 965

Regan, M., Sheth, K., \& Vogel, S. N. 1999, ApJ, 526, 97

Regan, M., Sheth, K., Teuben, P. J., et al. 2002, ApJ, 574, 126

Reshetnikov, V., \& Combes, F. 1998, A\&A, 337, 9

Reuter, H.-P., Sievers, A. W., Pohl, M., et al. 1996, A\&A, 306, 721

Rots, A. H. 1978, AJ, 83, 219

Saha, A., Sandage, A., Tammann, G. A., et al. 1999, ApJ, 522, 802

Sakamoto, K., Baker, A. J., \& Scoville, N. Z. 2000, ApJ, 533, 149

Salo, H., Rautiainen, P., Buta, R., et al. 1999, AJ, 117, 792

Sempere, M. J., Garcia-Burillo, S., Combes, F., et al. 1995, A\&A, 296, 45

Sheth, K., Vogel S. N., Regan, M., et al. 2002, AJ, 124, 2581

Smith, B. J., Harvey, P. M., Colome, C., et al. 1994, ApJ, 425, 91

Soida, M., Urbanik, M., Beck, R., et al. 2001, A\&A, 378, 40

Thornley, M., Regan, M., Helfer, T., et al. 1999, Ap\&SS, 269, 391

Toomre, A. 1977, Rev. Lect. at IAU Symp., 77

Zhang, X., Wright, M., \& Alexander, P. 1993, ApJ, 418, 100 